In phakic patients, cataract surgery was done in the same sitting prior to RD surgery. Authors should have mentioned the method of IOL power calculation. In our experience it is better to pass the buckle before phacoemulsification and intraocular lens (IOL) implantation as maneuvering during buckling may cause anterior chamber instability and IOL decentration. Surgeries were done by multiple surgeons. Different surgeons have different approach for a case so it would have affected the decision for preferring one surgical technique over the other. Also, it would have been more informative if the criteria for preferring surgical technique would have been mentioned.

Authors have preferred 5000cS silicon oil in all cases with oil removal at 3 months. High viscosity silicon oils are preferred in cases where long term or permanent tamponade is required [2]. Moreover, 5000cS oil is lighter than water, thus inflammatory cytokines get settled in inferior unsupported space leading to inferior PVR changes [3]. So, if inferior tamponade was the purpose of preferring $5000 \mathrm{cS}$, then heavy oils would have been better choice [4].

Authors have done regression analysis for finding correlation of seven factors with recurrence of RD. In multivariate regression, testing too many variables for the small sample size will overestimate associations.

Causes of higher IOP and better visual acuity in buckle group should have been discussed. Subgroup analysis for the grades of PVR would have been more informative regarding dealing with severe PVR. We appreciate authors for their choice of doing photocoagulation of bare choroid in cases of RR. Once again we applaud authors for sharing their experience.

\section{Compliance with ethical standards}

Conflict of interest The authors declare that they have no conflict of interest.

Publisher's note: Springer Nature remains neutral with regard to jurisdictional claims in published maps and institutional affiliations.

\section{References}

1. Eleinen KG, Mohalhal AA, Ghalwash DA et al. Vitrectomy with scleral buckling versus with inferior retinectomy in treating primary rhegmatogenous retinal detachment with PVR and inferior breaks. Eye. 2018.16:1.

2. Barca F, Caporossi T, Rizzo S. Silicone oil: different physical proprieties and clinical applications. BioMed research international. 2014;2014:502143.

3. de Juan E, Jr, McCuen B, Tiedeman J. Intraocular tamponade and surface tension. Surv Ophthalmol. 1985;30(1):47-51.

4. Joussen AM, Rizzo S, Kirchhof B et al. Heavy silicone oil versus standard silicone oil in as vitreous tamponade in inferior PVR (HSO Study): interim analysis. Acta Ophthalmol. 2011;89:e483-9.

\title{
Vitrectomy with scleral buckling versus with inferior retinectomy in treating primary rhegmatogenous retinal detachment with PVR and inferior breaks
}

\author{
Khaled G. Abu Eleinen ${ }^{1}{ }^{1} \cdot$ Ahmed A. Mohalhal ${ }^{1}$ - Dalia A. Ghalwash ${ }^{1} \cdot$ Ahmed A. Abdel-Kader $^{1}$.

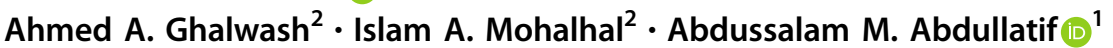

Received: 29 November 2018 / Accepted: 29 November 2018 / Published online: 19 December 2018

(c) The Royal College of Ophthalmologists 2018

We would like to thank Awasthi et al. [1] for their interest in our paper and their insightful comments.

Abdussalam M. Abdullatif

abdussalamabdullatif@kasralainy.edu.eg

1 Department of Ophthalmology, Kasr El Aini Hospital, Cairo University, El-Manial, Cairo, Egypt

2 Research Institute of Ophthalmology, Ministry of High Education, Giza, Egypt
Phacoemulsification with three-piece intraocular lens (IOL) implantation was done in all phakic patients as we believe that removing the lens gives access to the ora and far retinal periphery and allows dealing with the anterior proliferative vitreoretinopathy (PVR). The axial length was measured using optical biometry (IOL Master; Carl Zeiss, Oberkochen, Germany). If the axial length measured by optical biometry was shorter than that of the other eye, the axial length measurement was verified with A-scan ultrasonography [2]. SRK/T formula with the manufacturer's 
recommended A-constant was used to calculate IOL power. The axial length adjustment with Wang-Koch modification was applied. The refractive value in the other eye determined the refractive aim in the operated eye.

In the Buckle group, 360 degrees encircling silicone band was inserted through four scleral tunnels at the beginning of surgery before phacoemulsification or inserting any trocars. The surgeries were done by two groups of surgeons according to their surgical preference, the first group adopted vitrectomy combined with scleral buckle and the second group adopted vitrectomy with retinectomy. Baseline characteristics of both groups were not statistically different which indicate that both groups were similar without any bias towards any of the two groups.

Heavy Silicon Oil Study which compares heavy and standard silicone oil (SO) in patients with inferior PVR failed to demonstrate superiority of a heavy tamponade [3]. Moreover, several complications have been associated with heavy SO surgery, such as prolonged intraocular inflammation and intraocular pressure increase, probably due to the early emulsification of heavy SO [4]. That is why we preferred to use $\mathrm{SO}(5000 \mathrm{cs})$ as a tamponading agent which has the least rate of emulsification [5].

The mean postoperative IOP was significantly higher in the Buckle group throughout the whole follow-up period. This may be due to impaired venous drainage from the vortex veins, leading to congestion of the ciliary body. The edematous ciliary body is displaced anteriorly, shifting the lens-iris diaphragm forward and resulting in narrowing of the angle [6]. Visual acuity was better at first month in the Buckle group, but this difference disappeared throughout the remaining followup period, achieving the same functional outcome.
The number of patients prevented subgroup analysis for the grades of PVR. Performing a prospective larger study for better statistical analysis will be a great idea. Once again, we would like to thank Awasthi et al. for sharing their comments.

\section{Compliance with ethical standards}

Conflict of interest The authors declare that they have no conflict of interest.

\section{References}

1. Awasthi U, Grover R, Videkar C, Varshney A. Comment on "Vitrectomy with scleral buckling versus with inferior retinectomy in treating primary rhegmatogenous retinal detachment with PVR and inferior breaks". Eye. 2018; epub ahead of print 18 December 2018.

2. Rahman R, Bong CX, Stephenson J. Accuracy of intraocular lens power estimation in eyes having phacovitrectomy for rhegmatogenous retinal detachment. Retina. 2014;34:1415-20.

3. Joussen AM, Rizzo S, Kirchhof B, Schrage N, Li X, Lente C, et al. Heavy silicone oil versus standard silicone oil in as vitreous tamponade in inferior PVR (HSO Study): interim analysis. Acta Ophthalmol. 2011;89:e483-9.

4. Theelen T, Tilanus MAD, Klevering BJ. Intraocular inflammation following endotamponade with high-density silicone oil. Graefes Arch Clin Exp Ophthalmol. 2004;242:617-20.

5. Zafar S, Shakir M, Mahmood SA, Amin S, Iqbal Z. Comparison of 1000-Centistoke versus 5000-Centistoke Silicone Oil in complex retinal detachment surgery. J Coll Physicians Surg Pak. 2016;26:36-40.

6. Gedde SJ. Management of glaucoma after retinal detachment surgery. Curr Opin Ophthalmol. 2002;13:103-9.

\title{
Smartphone adaptor use for nasal endoscopy
}

\author{
Jerome How Ing $\mathrm{Ha}^{1} \cdot$ Suresh Reddy Sagili ${ }^{1}$
}

Received: 11 December 2018 / Accepted: 19 December 2018 / Published online: 16 January 2019

(c) The Royal College of Ophthalmologists 2019

Supplementary information The online version of this article (https:// doi.org/10.1038/s41433-018-0334-6) contains supplementary material, which is available to authorized users.

Jerome How Ing Ha

dr.jeromeha@gmail.com

1 Department of Ophthalmology, Royal Shrewsbury Hospital, Mytton Oak Road, SY3 8XQ Shrewsbury, UK
Nasal endoscopic examination is an important part of the preoperative assessment in patients presenting with nasolacrimal duct obstruction, particularly when planning endoscopic dacryocystorhinostomy (DCR). Deviated nasal septum can impede the access to the middle meatus and identifying this preoperatively facilitates surgical planning for simultaneous septoplasty where indicated. Other pathologies such as synechia, nasal polyps and chronic 